\title{
RETRACTION NOTE TO: Effect of Anti-Sticking Nanostructured Surface Coating on Minimally Invasive Electrosurgical Device in Brain
}

\author{
Han-Yi Cheng, ${ }^{1,2,3}$ Keng-Liang Ou, ${ }^{1,2,3,4}$ Hsi-Jen Chiang, ${ }^{1,2,4,5}$ and Li-Hsiang Lin ${ }^{2,5}$
}

${ }^{1}$ Graduate Institute of Biomedical Materials and Tissue Engineering, Taipei Medical University, Taipei 110, Taiwan; ${ }^{2}$ Research Center for Biomedical Devices and Prototyping Production, Taipei Medical University, Taipei, Taiwan; ${ }^{3}$ Research Center for Biomedical Implants and Microsurgery Devices, Taipei Medical University, Taipei 110, Taiwan; ${ }^{4}$ Department of Dentistry, Taipei Medical University-Shuang Ho Hospital, Taipei 235, Taiwan; and ${ }^{5}$ School of Dentistry, College of Oral Medicine, Taipei Medical University, Taipei 110, Taiwan

\section{RETRACTION NOTE:}

Annals of Biomedical Engineering, Vol. 43, No. 10, October 2015 pp. 2383-2393

DOI: $10.1007 / \mathbf{s 1 0 4 3 9 - 0 1 5 - 1 3 0 4 - 9}$

The article "Effect of Anti-Sticking Nanostructured Surface Coating on Minimally Invasive Electrosurgical Device in Brain", by Han-Yi Cheng, Keng-Liang Ou, Hsi-Jen Chiang, and Li-Hsiang Lin, published 8 April 2015 in the Annals of Biomedical Engineering, DOI: 10.1007/s10439-015-1304-9 has been retracted at the request of the Editor-in-Chief and the Biomedical Engineering Society per the Committee on Publication Ethics guidelines. The article shows evidence of text duplication, without a proper acknowledgement, from the following articles.

"Biomedical electrosurgery devices containing nanostructure for minimally invasive surgery: reduction of thermal injury and acceleration of wound healing for liver cancer", published 29 January 2015 in Journal of Materials Science: Materials in Medicine,
DOI: 10.1007/s10856-015-5416-4, by Wen-Tien Hsiao, Li-Hsiang Lin, Hsi-Jen Chiang, Keng-Liang Ou, HanYi Cheng.

"Research of electrosurgical unit with novel antiadhesion composite thin film for tumor ablation: Microstructural characteristics, thermal conduction properties, and biological behaviors", published 3 February 2015 in Journal of Biomedical Materials Research Part B: Applied Biomaterials, DOI: 10.1002/ jbm.b.33363 by Yun-Dun Shen, Li-Hsiang Lin, HsiJen Chiang, Keng-Liang Ou, Han-Yi Cheng.

In addition, the article is showing similarities with the following article which was submitted within a close timeframe:

"The Application of Advanced Nanostructured Film in Electrosurgical Device: Anti-Sticking Behavior and Thermal Injury", published 15 May 2015 in Journal of Nanomedicine \& Nanotechnology, DOI: $10.4172 / 2157-7439.1000291$ by Keng-Liang $\mathrm{Ou}$ and Han-Yi Cheng. Date received: March 30, 2015.

Address correspondence to Li-Hsiang Lin, School of Dentistry, College of Oral Medicine, Taipei Medical University, Taipei 110, Taiwan. Electronic mail: chytmu@gmail.com, lisa@tmu.edu.tw

This article has been retracted by the editor and publisher of the Annals of Biomedical Engineering as it has been concluded that portions of text in the article are either unoriginal or incorrectly cited and so the decision has been taken to remove this article from the scholarly field. It should no longer be cited with reference to publication in the Annals of Biomedical Engineering. 